\title{
Periprocedural dabigatran anticoagulation for atrial fibrillation ablation: do we have enough information to make a rational decision
}

\author{
Amit Noheria $•$ Samuel J. Asirvatham
}

Received: 14 June 2013 / Accepted: 27 June 2013 / Published online: 28 July 2013

(C) Springer Science+Business Media New York 2013

Atrial fibrillation (AF) ablation has become an established therapy for treating patients with drug-resistant symptomatic arrhythmia. Initial enthusiasm has been tempered by modest efficacy, frequent recurrences, and uncommon but significant periprocedural complications, including bleeding and systemic thromboembolization [1]. Additionally, AF ablation is a quality-of-life improving treatment with no demonstrated direct survival benefit. As a result, an exceptionally high value on procedural safety is critical to justify its continued utilization. Substantial advances have been made in reducing embolic complications [1-4]. These include intracardiac echocardiography (ICE) and heparinization with full anticoagulation prior to transseptal puncture $[2,3]$. A major advance has been the acceptance of uninterrupted therapeutic warfarin instead of routine periprocedural bridging with low-molecular weight heparin (LMWH). This has been associated with a significant reduction in embolic events without increasing the rate of vascular and bleeding complications [5].

The anticoagulation landscape for patients with nonvalvular AF has changed in the last 3-4 years with the adoption of direct thrombin inhibitor dabigatran and anti-factor Xa agents rivaroxaban and apixaban. Patients on dabigatran considered for AF ablation pose a dilemma for periprocedural management with three potential options. First, continue uninterrupted dabigatran with increased risk of bleeding on concomitant unfractionated heparin (UFH) during the procedure. Second, hold dabigatran for one to two doses before the procedure and restart postprocedure, a strategy enabled by the short half-life and a rapid onset of action. And third is the

A. Noheria $\cdot$ S. J. Asirvatham $(\bowtie)$

Division of Cardiovascular Diseases, Mayo Clinic College of

Medicine, 200 First Street SW, Rochester, MN 55905, USA

e-mail: asirvatham.samuel@mayo.edu

S. J. Asirvatham

Department of Pediatrics and Adolescent Medicine,

Mayo Clinic, Rochester, MN, USA transition from dabigatran to warfarin as the periprocedural anticoagulant with the inherent logistical challenges in accomplishing a therapeutic INR. A substudy of the RE-LY trial showed that dabigatran could be safely continued periprocedurally for direct current cardioversion with the risk of systemic embolism being similar to warfarin [6]. However, there are no randomized trial data to support use of dabigatran as the periprocedural anticoagulant for ablation.

There is some concern in using dabigatran with $\mathrm{AF}$ ablation because of the possibility of inadequate anticoagulation due to the short half-life, variable clearance, and the absence of a good test to monitor therapeutic effect. On the other hand, dabigatran is susceptible to drug-drug interactions and it intensifies the effect of UFH on activated partial thromboplastin time in vitro even when compared to factor Xa inhibitors [7]. Concomitant use of dabigatran with intraprocedural UFH may thus increase risk of bleeding unless it is held 1-2 days in advance [8]. Despite these challenges, many operators have adopted the strategy of performing AF ablation with brief interruption of dabigatran and resumption of dosing soon afterwards.

In this issue, Steinberg et al. report a meta-analysis of 10 observational studies, and thus summarize the hitherto published experience on periprocedural anticoagulation with dabigatran versus warfarin for AF ablation [9]. They show an overall safe profile of dabigatran similar to the published rates of complications in general [10]-10 of 1,501 patients $(0.7 \%)$ reportedly had any neurological event, and 24 $(1.6 \%)$ had major bleeding. Compared to warfarin, bleeding with dabigatran was similar but there was an absolute increase in neurological events by $0.47 \%$ (95\% confidence interval 0.07 and $0.99 \%$ ).

The authors deserve praise for compiling this metaanalysis. As the number of adverse events reported from individual studies is extremely small, a meta-analysis is a justifiable and valid way to obtain statistical power for any comparative assessments. Historically, meta-analyses have had the greatest impact on clinical practice when rare adverse 
events from multiple studies have been combined to provide meaningful comparisons in large numbers of patients [11].

The results of this review need to be interpreted in context of limitations as pointed out by the authors, a meta-analysis being only as good as the component studies. The analysis is constrained by the overall quality, number, and size of included studies; underscored by four unpublished studies included in the abstract form (although Ellis et al. [12] has now been published) and the observational, often retrospective, study designs. Most studies included unselected unmatched controls and unadjusted event rates from individual studies have been pooled in the meta-analysis without adjustment for confounders. It is plausible that patients on dabigatran were in general a healthier subset and a randomized head-to-head comparison would have revealed a greater benefit in favor of warfarin (or vice versa).

The authors of the meta-analysis do not provide forest plots to visually depict the variability of results in the component studies, nor show any objective assessment of heterogeneity. Further, meta-regression to explain heterogeneity by study characteristics is not presented though it probably could not have been performed because of limited statistical power. Similarly, assessment of publication bias has not been presented. There are obvious methodological dissimilarities among the studies included in the meta-analysis, with differences in the timing of holding and restarting dabigatran for the procedure, ablation techniques, types of catheters, and target activated clotting times (ACT). Further, among controls, LMWH bridging was used instead of uninterrupted warfarin in three unpublished studies. Comparing only uninterrupted warfarin versus dabigatran by excluding these three studies, the reduction in neurologic events with warfarin was surprisingly attenuated and lost statistical significance. The exclusion of studies using an inferior warfarin strategy with interruption of anticoagulation would have been expected to reveal a larger signal in favor of warfarin [5]. This unexpected result is explained by chance variation due to small number of events, and other differences in methodology and confounding in the excluded studies. As an example, the abstract by Ellis et al. was identified as having a control strategy of suspending warfarin and bridging with LMWH but the recently published manuscript actually shows that $63 \%$ of the controls had their procedure on therapeutic warfarin [12]. Another small observational study published after conducting this meta-analysis showed similar stroke and bleeding rates with dabigatran as with interruption of warfarin and bridging with LMWH [13].

There was a marked increase in the relative risk of pericardial tamponade with dabigatran (6\%) compared to warfarin $(1 \%)$ in the multicenter study by Lakkireddy et al. [14]. Based on this study, it has been proposed that holding just the morning dose of dabigatran might not be adequate and a longer period of interruption is required [8]. In the present meta-analysis, most studies continued dabigatran till the night before the procedure and only held the dose on the morning of the procedure. Maddox et al. [15] ablated with no interruption in dabigatran at all, without any increase in bleeding compared to warfarin. Thus in patients with normal renal function, it might be reasonable to hold only the morning dose of dabigatran just prior to the procedure. The remarkably increased risk of tamponade seen by Lakkireddy et al. might however be related to their administering dabigatran dose immediately post-ablation $(0-3 \mathrm{~h})$, although, Kim et al. show that it might be safe to restart dabigatran as early as $4 \mathrm{~h}$ after the procedure [16].

Prior studies have suggested that targeting a higher ACT level of 350-400 s during ablation might reduce thromboembolic events, especially in patients with spontaneous echocontrast [17]. However, in the case of a therapeutic INR, this may lead to an excess of bleeding events as observed in the studies by Maddox et al. [15] and Bassiouny et al. [18]. Contemporary studies with open irrigation tip catheters suggest an ACT level even below $250 \mathrm{~s}$ might be adequate for prevention of thromboembolism, and is associated with reduction in bleeding complications [19].

The competing complications associated with anticoagulation use (too little), thromboembolism, and too much (bleeding), are partially addressed with adjunctive non-anticoagulation strategies. Routine ultrasound (SonoSite, Bothell, Washington) for vascular access decreases vascular complications despite anticoagulation. Open irrigation catheters [2] and novel techniques that potentially decrease coagulum on the ablation electrode tissue interface despite heparin use in turn [20] may allow less embolic risk with more modest levels of anticoagulation.

None of the studies included in the meta-analysis assessed the incidence of new brain lesions with ablation as detected by MRI. Although subclinical brain embolisms may be minor and reversible in some patients [21], and surely not as dreadful as a manifest stroke, they potentially have longterm deleterious effects. New cerebral lesions are detectable by MRI in over a third of patients who are off warfarin and undergo AF ablation with non-irrigated radiofrequency catheters [22], though the risk is lower with uninterrupted warfarin and open irrigated-tip catheters [21-24]. A prospective study looking for subclinical cerebral lesions will be very valuable in assessing the safety of dabigatran.

Apart from dabigatran, data on factor Xa inhibitors are also being generated. A recent abstract from a small multicenter series showed safety and efficacy of AF ablation with periprocedural rivaroxaban [25]. Rivaroxaban was held on the day of procedure and restarted $6 \mathrm{~h}$ postprocedure, with risk of bleeding and TIA similar to uninterrupted warfarin.

To summarize, in selected patients anticoagulation with dabigatran around the time of $\mathrm{AF}$ ablation has low rates of neurologic and bleeding complications and may be a reasonable strategy, especially in patients already on the drug. Any small 
absolute benefit of uninterrupted warfarin over dabigatran might be mitigated by the logistical challenges in transitioning patients to a therapeutic INR with warfarin. For most patients, no more than one dose of dabigatran needs to be withheld prior to the procedure, and resumption of dabigatran 4-6h after procedure seems to be safe. In patients undergoing AF ablation on uninterrupted anticoagulation, a lower ACT goal with use of an open tip-irrigation catheter offers adequate prevention of stroke. Additionally, heightened vigilance for thrombus and coagulum formation with intraprocedural ICE is useful regardless of the anticoagulation strategy.

\section{References}

1. Calkins, H., Kuck, K. H., Cappato, R., Brugada, J., Camm, A. J., Chen, S. A., et al. (2012). $2012 \mathrm{hrs} / \mathrm{ehra} / \mathrm{ecas}$ expert consensus statement on catheter and surgical ablation of atrial fibrillation: recommendations for patient selection, procedural techniques, patient management and follow-up, definitions, endpoints, and research trial design. Journal of Interventional Cardiac Electrophysiology, 33, 171-257.

2. Asirvatham, S. J. (2007). Ablation for atrial fibrillation: can we decrease thromboembolism without increasing the risk for bleeding? Circulation, 116, 2517-2519.

3. Bruce, C. J., Friedman, P. A., Narayan, O., Munger, T. M., Hammill, S. C., Packer, D. L., et al. (2008). Early heparinization decreases the incidence of left atrial thrombi detected by intracardiac echocardiography during radiofrequency ablation for atrial fibrillation. Journal of Interventional Cardiac Electrophysiology, 22, 211-219.

4. Asirvatham, S. J., Bruce, C. J., \& Friedman, P. A. (2003). Advances in imaging for cardiac electrophysiology. Coronary Artery Disease, 14, 3-13.

5. Santangeli, P., Di Biase, L., Horton, R., Burkhardt, J. D., Sanchez, J., Al-Ahmad, A., et al. (2012). Ablation of atrial fibrillation under therapeutic warfarin reduces periprocedural complications: evidence from a meta-analysis. Circulation: Arrhythmia and Electrophysiology, 5, 302-311.

6. Nagarakanti, R., Ezekowitz, M. D., Oldgren, J., Yang, S., Chernick, M., Aikens, T. H., et al. (2011). Dabigatran versus warfarin in patients with atrial fibrillation: an analysis of patients undergoing cardioversion. Circulation, 123, 131-136.

7. Walenga, J. M., \& Adiguzel, C. (2010). Drug and dietary interactions of the new and emerging oral anticoagulants. International Journal of Clinical Practice, 64, 956-967.

8. Knight, B. P. (2012). Anticoagulation for atrial fibrillation ablation: what is the optimal strategy? Journal of the American College of Cardiology, 59, 1175-1177.

9. Steinberg, B. A., Hasselblad, V., Atwater, B. D., Bahnson, T. D., Washam, J. B., Alexander, J. H., et al. (2013). Dabigatran for periprocedural anticoagulation following radiofrequency ablation for atrial fibrillation: a meta-analysis of observational studies. Journal of Interventional Cardiac Electrophysiology (this issue)

10. Cappato, R., Calkins, H., Chen, S. A., Davies, W., Iesaka, Y., Kalman, J., et al. (2010). Updated worldwide survey on the methods, efficacy, and safety of catheter ablation for human atrial fibrillation. Circulation: Arrhythmia and Electrophysiology, 3, 32-38.

11. Coplen, S. E., Antman, E. M., Berlin, J. A., Hewitt, P., \& Chalmers, T. C. (1990). Efficacy and safety of quinidine therapy for maintenance of sinus rhythm after cardioversion. A meta-analysis of randomized control trials. Circulation, 82, 1106-1116.
12. Kaiser DW, Streur MM, Nagarakanti R, Whalen SP, Ellis CR (2013) Continuous warfarin versus periprocedural dabigatran to reduce stroke and systemic embolism in patients undergoing catheter ablation for atrial fibrillation or left atrial flutter. Journal of Interventional Cardiac Electrophysiology.

13. Imamura, K., Yoshida, A., Takei, A., Fukuzawa, K., Kiuchi, K., Takami, K., et al. (2013). Dabigatran in the peri-procedural period for radiofrequency ablation of atrial fibrillation: efficacy, safety, and impact on duration of hospital stay. Journal of Interventional Cardiac Electrophysiology.

14. Lakkireddy, D., Reddy, Y. M., Di Biase, L., Vanga, S. R., Santangeli, P., Swarup, V., et al. (2012). Feasibility and safety of dabigatran versus warfarin for periprocedural anticoagulation in patients undergoing radiofrequency ablation for atrial fibrillation: results from a multicenter prospective registry. Journal of the American College of Cardiology, 59, 1168-1174.

15. Maddox, W., Kay, G. N., Yamada, T., Osorio, J., Doppalapudi, H., Plumb, V. J., et al. (2013). Dabigatran versus warfarin therapy for uninterrupted oral anticoagulation during atrial fibrillation ablation. Journal of Cardiovascular Electrophysiology, 24(8), 861-865.

16. Kim, J. S., She, F., Jongnarangsin, K., Chugh, A., Latchamsetty, R., Ghanbari, H., et al. (2013). Dabigatran vs warfarin for radiofrequency catheter ablation of atrial fibrillation. Heart Rhythm, 10, 483489.

17. Ren, J. F., Marchlinski, F. E., Callans, D. J., Gerstenfeld, E. P., Dixit, S., Lin, D., et al. (2005). Increased intensity of anticoagulation may reduce risk of thrombus during atrial fibrillation ablation procedures in patients with spontaneous echo contrast. Journal of Cardiovascular Electrophysiology, 16, 474-477.

18. Bassiouny, M., Saliba, W., Rickard, J., Shao, M., Sey, A., Diab, M., et al. (2013). Use of dabigatran for periprocedural anticoagulation in patients undergoing catheter ablation for atrial fibrillation. Circulation: Arrhythmia and Electrophysiology, 6, 460-466.

19. Winkle, R. A., Mead, R. H., Engel, G., \& Patrawala, R. A. (2011). Safety of lower activated clotting times during atrial fibrillation ablation using open irrigated tip catheters and a single transseptal puncture. The American Journal of Cardiology, 107, 704-708.

20. Lim, B., Venkatachalam, K. L., Jahangir, A., Johnson, S. B., \& Asirvatham, S. J. (2008). Concurrent application of charge using a novel circuit prevents heat-related coagulum formation during radiofrequency ablation. Journal of Cardiovascular Electrophysiology, 19, 843-850.

21. Deneke, T., Shin, D. I., Balta, O., Bunz, K., Fassbender, F., Mugge, A., et al. (2011). Postablation asymptomatic cerebral lesions: longterm follow-up using magnetic resonance imaging. Heart Rhythm, $8,1705-1711$.

22. Gaita, F., Leclercq, J. F., Schumacher, B., Scaglione, M., Toso, E., Halimi, F., et al. (2011). Incidence of silent cerebral thromboembolic lesions after atrial fibrillation ablation may change according to technology used: comparison of irrigated radiofrequency, multipolar nonirrigated catheter and cryoballoon. Journal of Cardiovascular Electrophysiology, 22, 961-968.

23. Herrera Siklody, C., Deneke, T., Hocini, M., Lehrmann, H., Shin, D. I., Miyazaki, S., et al. (2011). Incidence of asymptomatic intracranial embolic events after pulmonary vein isolation: comparison of different atrial fibrillation ablation technologies in a multicenter study. Journal of the American College of Cardiology, 58, 681688.

24. Madhavan, M., Govil, S. R., \& Asirvatham, S. J. (2012). Signals. Circulation: Arrhythmia and Electrophysiology, 5, 2-4.

25. Lakkireddy, D., Reddy, M., Swarup, V., Baqdunes, M. W., Mansour, M., Chaloub, F., et al. (2013). Uninterrupted rivaroxaban vs. warfarin for periprocedural anticoagulantion during atrial fibrillation ablation: a multicenter experience (abstract). Heart Rhythm, 10, 41-95. 